\title{
Contact real hypersurfaces in the complex hyperbolic quadric
}

\author{
Sebastian Klein ${ }^{1}$ (D) Young Jin Suh ${ }^{2}$
}

Received: 8 June 2018 / Accepted: 18 January 2019 / Published online: 1 February 2019 (c) Fondazione Annali di Matematica Pura ed Applicata and Springer-Verlag GmbH Germany, part of Springer Nature 2019

\begin{abstract}
We discuss the geometry of contact real hypersurfaces with constant mean curvature in the complex hyperbolic quadric $Q^{m *}=\mathrm{SO}_{m, 2}^{o} / \mathrm{SO}_{m} \mathrm{SO}_{2}$, where $m \geq 3$. These hypersurfaces were classified in Berndt and Suh (Proc Am Math Soc 143:2637-2649, 2015), and we study the individual types (two types of tubes around totally geodesic submanifolds of $Q^{m *}$ and one type of horosphere) that have been found in that classification.
\end{abstract}

Keywords Contact hypersurface $\cdot$ Kähler structure $\cdot$ Complex conjugation · Complex hyperbolic quadric

Mathematics Subject Classification Primary 53C40 - Secondary 53C55

\section{Introduction}

Following Sasaki [19] and Okumura [13], an odd-dimensional, smooth manifold $M^{2 m-1}$ is called an almost contact manifold if the structure group of its tangent bundle can be reduced to $U_{m-1} \times 1$ (where $U_{m-1}$ refers to the natural real representation of the unitary group in $m-1$ complex variables). $M^{2 m-1}$ is called a contact manifold if there exists a smooth 1 -form $\eta$ on $M^{2 m-1}$ so that $\eta \wedge d \eta^{m-1} \neq 0$; such an $\eta$ is then called a contact form on $M^{2 m-1}$.

It was shown by Sasaki [19, Theorem 5] that $M^{2 m-1}$ is an almost contact manifold if and only if there exists an almost contact metric structure $(\phi, \xi, \eta, g)$ on $M^{2 m-1}$. Here $\phi$ is an

Young Jin Suh was supported by Grant Project No. NRF-2018-R1D1A1B-05040381 from National Research Foundation of Korea.

Sebastian Klein

s.klein@math.uni-mannheim.de

Young Jin Suh

yjsuh@knu.ac.kr

1 Fakultät für Wirtschaftsinformatik und Wirtschaftsmathematik, Universität Mannheim, Seminargebäude A5, 68131 Mannheim, Germany

2 Department of Mathematics \& RIRCM, College of Natural Sciences, Kyungpook National University, Daegu 41566, Republic of Korea 
endomorphism field on $M^{2 m-1}, \xi$ is a vector field on $M^{2 m-1}, \eta$ is a 1-form on $M^{2 m-1}$ and $g$ is a Riemannian metric on $M^{2 m-1}$, and these data are related to each other in the following way: First we have

$$
\phi^{2} X=-X+\eta(X) \xi, \quad \phi(\xi)=0, \quad \eta(\phi X)=0, \quad \eta(\xi)=1
$$

for all vector fields $X$ on $M^{2 m-1}$, meaning that $(\phi, \xi, \eta)$ is an almost contact structure, and moreover this structure is adapted to the Riemannian metric $g$ by

$$
g(\phi X, \phi Y)=g(X, Y)-\eta(X) \eta(Y) \quad \text { and } \quad \eta(X)=g(X, \xi)
$$

for all vector fields $X, Y$ on $M^{2 m-1}$.

Clearly, if $M^{2 m-1}$ has an almost contact metric structure $(\phi, \xi, \eta, g)$ so that $\eta \wedge d \eta^{m-1} \neq 0$ holds, then $M^{2 m-1}$ is a contact manifold. Conversely, if $M^{2 m-1}$ is a contact manifold, then for any contact form $\eta$ on $M^{2 m-1}$ there exists an almost contact metric structure $(\phi, \xi, \eta, g)$ with this $\eta$ by a result due to Sasaki [19, Theorem 4].

Let us now consider a real hypersurface $M$ of a Kähler manifold $\bar{M}$ of complex dimension $m$. Then $M$ has real dimension $2 m-1$, and the complex structure $J$ and the Riemannian metric $g$ of $\bar{M}$ induce an almost contact metric structure $(\phi, \xi, \eta, g)$ on $M$ : Let $N$ be a unit normal vector field of $M$ in $\bar{M}$, then we choose $\phi$ as the structure tensor field defined by letting $\phi X$ be the $M$-tangential part of $J X$ for any $X \in T M$, choose $\xi$ as the Reeb vector field $\xi=-J N$, and choose $\eta=g(\cdot, \xi)$. If there exists a smooth, everywhere nonzero function $\rho$ on $M$ in this setting so that

$$
\mathrm{d} \eta(X, Y)=\rho \cdot g(\phi X, Y)
$$

holds for all vector fields $X, Y$ on $M$, then $M$ is a contact manifold, and $\eta$ a contact form on $M$. In this case $M$ is called a contact hypersurface of $\bar{M}$, see also Blair [6], Dragomir and Perrone [7]. It was noted by Okumura [13, Eq. (2.13)] that the condition (1.1) is equivalent to

$$
S \phi+\phi S=k \cdot \phi,
$$

where $S$ denotes the shape operator of the hypersurface $M$ in $\bar{M}$ with respect to the unit normal vector field $N$, and $k=2 \rho$. If the complex dimension of $\bar{M}$ is at least 3 in this setting, then the function $\rho$ resp. $k$ is necessarily constant, see [4, Proposition 2.5].

Pursuant to these ideas, the contact hypersurfaces have been classified in the Hermitian symmetric spaces of rank 1, namely in the complex projective space $\mathbb{C} P^{m}$ and its noncompact dual, the complex hyperbolic space $\mathbb{C} H^{m}$. Yano and Kon showed in [24, Theorem VI.1.5] that a connected contact hypersurface with constant mean curvature of the complex projective space $\mathbb{C} P^{m}$ with $m \geq 3$ is locally congruent either to a geodesic hypersphere, or to a tube over a real projective space $\mathbb{R} P^{n}, m=2 n$, embedded in $\mathbb{C} P^{m}$ as a totally real, totally geodesic submanifold. Vernon proved in [23] that a complete, connected contact real hypersurface in $\mathbb{C} H^{m}$ with $m \geq 3$ is congruent to a tube around a totally geodesic $\mathbb{C} H^{m-1}$ in $\mathbb{C} H^{m}$, a tube around a real form $\mathbb{R} H^{m}$ in $\mathbb{C} H^{m}$, a geodesic hypersphere in $\mathbb{C} H^{m}$, or a horosphere in $\mathbb{C} H^{m}$. Note that all the contact hypersurfaces in $\mathbb{C} P^{m}$ or $\mathbb{C} H^{m}$ are homogeneous and therefore have constant principal curvatures, in particular constant mean curvature. We would like to mention that Pérez has carried out a nice investigation of certain real hypersurfaces in $\mathbb{C} P^{m}$ in [14].

When we consider more complicated Hermitian symmetric spaces as ambient space $\bar{M}$, there can be contact hypersurfaces which do not have constant mean curvature. The class of all contact hypersurfaces $M$ in $\bar{M}$ is very complicated, and a full classification does not appear to be feasible at the present time. However, if one considers only contact 
hypersurfaces $M$ with constant mean curvature, the classification problem becomes tractable at least when $\bar{M}$ is a Hermitian symmetric space of rank 2 .

The series of irreducible Hermitian symmetric spaces of rank 2 comprise the complex quadrics $Q^{m}=S_{m+2} / S_{2} S_{m}$ (isomorphic to the real 2-Grassmannians $G_{2}^{+}\left(\mathbb{R}^{m+2}\right.$ ) of oriented planes in $\left.\mathbb{R}^{m+2}\right)$, the complex 2-Grassmannians $G_{2}\left(\mathbb{C}^{m+2}\right)=S U_{m+2} / S\left(U_{2} U_{m}\right)$, and their non-compact duals, the complex hyperbolic quadrics $Q^{m *}=\mathrm{SO}_{2, m}^{o} / \mathrm{SO}_{2} \mathrm{SO}_{m}$ and the duals of the complex 2-Grassmannians $G_{2}^{*}\left(\mathbb{C}^{m+2}\right)=S U_{2, m} / S\left(U_{2} U_{m}\right)$. We would like to mention the very nice investigations by Pérez et al. of specific types of real hypersurfaces in $Q^{m}$ in [15] and in $G_{2}\left(\mathbb{C}^{m+2}\right)$ in [16] and [17].

The classification of contact hypersurfaces with constant mean curvature in the complex quadric $Q^{m}$ and in its non-compact dual, the complex hyperbolic quadric $Q^{m *}$ has been carried out by Berndt and the second author of the present paper in [4]. The result for $Q^{m *}$ is stated as Theorem A. For the case of the complex quadric $Q^{m}$, a different classification proof has been given in [22] by the second author of the present paper.

In the complex 2-Grassmannians $G_{2}\left(\mathbb{C}^{m+2}\right)$, the contact hypersurfaces with constant mean curvature have also been classified by the second author of the present paper in [21]. He shows that such a hypersurface is congruent to an open part of a tube around a totally geodesic quaternionic projective space $\mathbb{H} P^{n}$ in $G_{2}\left(\mathbb{C}^{m+2}\right)$, where $m=2 n$. For the non-compact dual $G_{2}^{*}\left(\mathbb{C}^{m+2}\right)$ of these Grassmannians, as far as we know there does not exist a classification of contact hypersurfaces with constant mean curvature. However, Berndt et al. [2] classified contact hypersurfaces of $G_{2}^{*}\left(\mathbb{C}^{m+2}\right)$ which satisfy another curvature condition, namely that the principal curvature function $\alpha$ corresponding to the Reeb vector field of the hypersurface is constant. The result of the classification is that any such hypersurface of $G_{2}^{*}\left(\mathbb{C}^{m+2}\right)$ is congruent either to an open part of a tube around a totally geodesic quaternionic hyperbolic space $\mathbb{H} H^{n}$ in $G_{2}\left(\mathbb{C}^{m+2}\right)$ (only if $m=2 n$ is even), or to an open part of a horosphere in a certain position in $G_{2}^{*}\left(\mathbb{C}^{m+2}\right)$. Note that all these hypersurfaces have constant mean curvature (this follows from [2, Propositions 4.1, 4.2(ii)]).

The purpose of the present paper is to study the local geometry of the contact real hypersurfaces with constant mean curvature in the complex hyperbolic quadric $Q^{m *}$. As mentioned above, those hypersurfaces were classified by Berndt and Suh. The result is as follows:

Theorem A (Berndt and Suh [4]) Let M be a connected orientable real hypersurface with constant mean curvature in the complex hyperbolic quadric $Q^{m *}, m \geq 3$. Then $M$ is a contact hypersurface if and only if $M$ is congruent to an open part of one of the following contact hypersurfaces in $Q^{m *}$ :

(i) the tube of radius $r>0$ around the complex hyperbolic quadric $Q^{m-1 *}$ which is embedded in $Q^{m *}$ as a totally geodesic complex hypersurface;

(ii) a horosphere in $Q^{m *}$ whose center at infinity is the equivalence class of an $\mathfrak{A}$-principal geodesic in $Q^{m *}$;

(iii) the tube of radius $r>0$ around the $n$-dimensional real hyperbolic space $\mathbb{R} H^{n}$ which is embedded in $Q^{m *}$ as a real space form of $Q^{m *}$.

We want to describe the local geometry of the three types (i)-(iii) of real hypersurfaces in $Q^{m *}$ given in the above theorem. For this purpose we first need to study the geometry of $Q^{m *}$ itself. In particular we need to describe the "fundamental geometric structures" of $Q^{m *}$; they are its Riemannian metric $g$, its Hermitian structure $J$ and a certain $S^{1}$-subbundle 
$\mathfrak{A}$ of $\operatorname{End}\left(T Q^{m *}\right)$ which can be used to characterize the orbits of the isotropy action on the tangent space of $Q^{m *}$. The terms " $\mathfrak{A}$-principal" which occurs in Theorem A refers to one of the two singular orbits of this action. For the complex quadric $Q^{m}$ the corresponding $S^{1}$ -bundle $\mathfrak{A}$ is obtained from the shape operator of the embedding $Q^{m} \hookrightarrow \mathbb{C} P^{m+1}$; it has first been introduced by Reckziegel in [18] and has many times been shown to be very useful for the investigation of the submanifold geometry of $Q^{m}$, for example for the classification of the totally geodesic submanifolds of $Q^{m}$ by the first author of the present paper in [9], and in several classifications of real hypersurfaces in $Q^{m}$ satisfying certain curvature conditions by the second author of the present paper and his coauthors. We would like to base our investigation of real hypersurfaces in $Q^{m *}$ on the analogous $S^{1}$-subbundle $\mathfrak{U}$ for $Q^{m *}$, which has, as far as we know, not before been described in full detail in the literature. The situation is more complicated for $Q^{m *}$ than for $Q^{m}$ here, because there does not exist a complex hypersurface embedding of $Q^{m *}$ into $\mathbb{C} H^{m+1}$, as was shown by Smyth [20], therefore $\mathfrak{A}$ for $Q^{m *}$ cannot be obtained in the analogous manner as for $Q^{m}$. Instead it needs to be obtained from the representation of $Q^{m *}$ as the quotient manifold $\mathrm{SO}_{2, m} / \mathrm{SO}_{2} \mathrm{SO}_{m}$ regarded as a non-compact Hermitian symmetric space. We thus carry out the description of the symmetric space structure of $Q^{m *}$ and of the mentioned fundamental geometric structures in Sect. 2.

The remainder of the paper is concerned with the construction of the contact hypersurfaces in $Q^{m *}$ that are given in Theorem A. In Sect. 3 we construct the tubes around totally geodesic submanifolds that occur in Theorem A(i),(iii). Section 4 recalls how horospheres in non-compact Riemannian symmetric spaces of rank 2 are constructed, and then a description of the horosphere of Theorem A(ii) is given.

\section{The complex hyperbolic quadric}

The $m$-dimensional complex hyperbolic quadric $Q^{m *}$ is the non-compact dual of the $m$-dimensional complex quadric $Q^{m}$, i.e. the simply connected Riemannian symmetric space whose curvature tensor is the negative of the curvature tensor of $Q^{m}$.

The complex hyperbolic quadric $Q^{m *}$ cannot be realized as a homogeneous complex hypersurface of the complex hyperbolic space $\mathbb{C} H^{m+1}$. In fact, Smyth [20, Theorem 3(ii)] has shown that every homogeneous complex hypersurface in $\mathbb{C} H^{m+1}$ is totally geodesic. This is in marked contrast to the situation for the complex quadric $Q^{m}$, which can be realized as a homogeneous complex hypersurface of the complex projective space $\mathbb{C} P^{m+1}$ in such a way that the shape operator for any unit normal vector to $Q^{m}$ is a real structure on the corresponding tangent space of $Q^{m}$, see [18] and [9]. Another related result by Smyth, [20, Theorem 1], which states that any complex hypersurface of $\mathbb{C} H^{m+1}$ for which the square of the shape operator has constant eigenvalues (counted with multiplicity) is totally geodesic, also precludes the possibility of a model of $Q^{m *}$ as a complex hypersurface of $\mathbb{C} H^{m+1}$ with the analogous property for the shape operator.

Therefore we realize the complex hyperbolic quadric $Q^{m *}$ as the quotient manifold $\mathrm{SO}_{2, m} / \mathrm{SO}_{2} \mathrm{SO}_{m}$. As $Q^{1^{*}}$ is isomorphic to the real hyperbolic space $\mathbb{R} H^{2}=S O_{1,2} / \mathrm{SO}_{2}$, and $Q^{2 *}$ is isomorphic to the Hermitian product of complex hyperbolic spaces $\mathbb{C} H^{1} \times \mathbb{C} H^{1}$, we suppose $m \geq 3$ in the sequel and throughout this paper. Let $G:=S O_{2, m}$ be the transvection group of $Q^{m *}$ and $\mathrm{K}:=\mathrm{SO}_{2} \mathrm{SO}_{m}$ be the isotropy group of $Q^{m *}$ at the "origin" $p_{0}:=e K \in Q^{m *}$. Then 


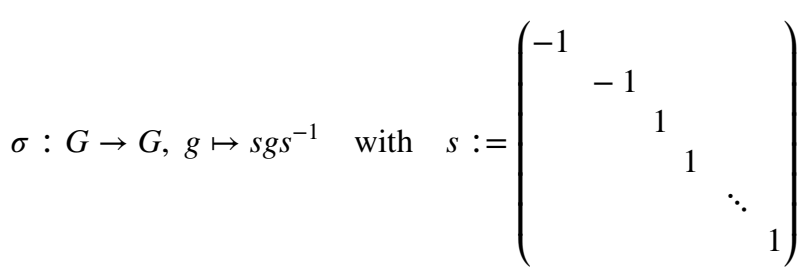

is an involutive Lie group automorphism of $G$ with $\operatorname{Fix}(\sigma)_{0}=K$, and therefore $Q^{m *}=G / K$ is a Riemannian symmetric space. The center of the isotropy group $\mathrm{K}$ is isomorphic to $\mathrm{SO}_{2}$, and therefore $Q^{m *}$ is in fact a Hermitian symmetric space.

The Lie algebra $\mathfrak{g}:=\mathfrak{S}_{2, m}$ of $G$ is given by

$$
\mathfrak{g}=\left\{X \in \mathfrak{g} \mathfrak{l}(m+2, \mathbb{R}) \mid X^{t} \cdot s=-s \cdot X\right\}
$$

(see [10, p. 59]). In the sequel we will write members of $\mathfrak{g}$ as block matrices with respect to the decomposition $\mathbb{R}^{m+2}=\mathbb{R}^{2} \oplus \mathbb{R}^{m}$, i.e. in the form

$$
X=\left(\begin{array}{ll}
X_{11} & X_{12} \\
X_{21} & X_{22}
\end{array}\right)
$$

where $X_{11}, X_{12}, X_{21}, X_{22}$ are real matrices of the dimension $2 \times 2,2 \times m, m \times 2$ and $m \times m$, respectively. Then

$$
\mathfrak{g}=\left\{\left(\begin{array}{ll}
X_{11} & X_{12} \\
X_{21} & X_{22}
\end{array}\right) \mid X_{11}^{t}=-X_{11}, X_{12}^{t}=X_{21}, X_{22}^{t}=-X_{22}\right\} .
$$

The linearization $\sigma_{L}=\operatorname{Ad}(s): \mathfrak{g} \rightarrow \mathfrak{g}$ of the involutive Lie group automorphism $\sigma$ induces the Cartan decomposition $\mathfrak{g}=\mathfrak{H} \oplus \mathfrak{m}$, where the Lie subalgebra

$$
\begin{aligned}
\mathfrak{f} & =\operatorname{Eig}\left(\sigma_{*}, 1\right)=\left\{X \in \mathfrak{g} \mid s X s^{-1}=X\right\} \\
& =\left\{\left(\begin{array}{cc}
X_{11} & 0 \\
0 & X_{22}
\end{array}\right) \mid X_{11}^{t}=-X_{11}, X_{22}^{t}=-X_{22}\right\} \cong \mathfrak{s o}_{2} \oplus \mathfrak{s}_{m}
\end{aligned}
$$

is the Lie algebra of the isotropy group $K$, and the $2 m$-dimensional linear subspace

$$
\mathfrak{m}=\operatorname{Eig}\left(\sigma_{*},-1\right)=\left\{X \in \mathfrak{g} \mid s X s^{-1}=-X\right\}=\left\{\left(\begin{array}{cc}
0 & X_{12} \\
X_{21} & 0
\end{array}\right) \mid X_{12}^{t}=X_{21}\right\}
$$

is canonically isomorphic to the tangent space $T_{p_{0}} Q^{m *}$. Under the identification $T_{p_{0}} Q^{m *} \cong \mathfrak{m}$, the Riemannian metric $g$ of $Q^{m *}$ (where the constant factor of the metric is chosen so that the formulae become as simple as possible) is given by

$$
g(X, Y)=\frac{1}{2} \operatorname{tr}\left(Y^{t} \cdot X\right)=\operatorname{tr}\left(Y_{12} \cdot X_{21}\right) \quad \text { for } \quad X, Y \in \mathfrak{m} .
$$

$g$ is clearly $\operatorname{Ad}(K)$-invariant and therefore corresponds to an $\operatorname{Ad}(G)$-invariant Riemannian metric on $Q^{m *}$. The complex structure $J$ of the Hermitian symmetric space is given by 


$$
J X=\operatorname{Ad}(j) X \quad \text { for } \quad X \in \mathfrak{m}, \quad \text { where } j:=\left(\begin{array}{ccccc}
0 & 1 & & & \\
-1 & 0 & & & \\
& 1 & & \\
& & 1 & & \\
& & & \ddots & \\
& & & & 1
\end{array}\right) \in K
$$

Because $j$ is in the center of $K$, the orthogonal linear map $J$ is $\operatorname{Ad}(K)$-invariant and thus defines an $\operatorname{Ad}(G)$-invariant Hermitian structure on $Q^{m *}$. By identifying the multiplication with the unit complex number $i$ with the application of the linear map $J$, the tangent spaces of $Q^{m *}$ thus become $m$-dimensional complex linear spaces, and we will adopt this point of view in the sequel.

Like for the complex quadric (again compare [18] and [9]), there is another important structure on the tangent bundle of the complex quadric besides the Riemannian metric and the complex structure, namely an $S^{1}$-bundle $\mathfrak{A}$ of real structures (conjugations). The situation here differs from that of the complex quadric in that for $Q^{m *}$, the real structures in $\mathfrak{A}$ cannot be interpreted as the shape operator of a complex hypersurface in a complex space form, but as the following considerations will show, $\mathfrak{A}$ still plays a fundamental role in the description of the geometry of $Q^{m *}$.

Let

$$
a_{0}:=\left(\begin{array}{cccccc}
1 & & & & & \\
& -1 & & & & \\
& & 1 & & & \\
& & & 1 & & \\
& & & & \ddots & \\
& & & & & 1
\end{array}\right)
$$

Note that we have $a_{0} \notin K$, but only $a_{0} \in O_{2} S O_{m}$. However, $\operatorname{Ad}\left(a_{0}\right)$ still leaves $\mathfrak{m}$ invariant and therefore defines an $\mathbb{R}$-linear map $A_{0}$ on the tangent space $\mathfrak{m} \cong T_{p_{0}} Q^{m *}$. $A_{0}$ turns out to be an involutive orthogonal map with $A_{0} \circ J=-J \circ A_{0}$ (i.e. $A_{0}$ is anti-linear with respect to the complex structure of $T_{p_{0}} Q^{m *}$ ), and hence a real structure on $T_{p_{0}} Q^{m *}$. But $A_{0}$ commutes with $\operatorname{Ad}(g)$ not for all $g \in K$, but only for $g \in S O_{m} \subset K$. More specifically, for $g=\left(g_{1}, g_{2}\right) \in K$ with $g_{1} \in S O_{2}$ and $g_{2} \in S O_{m}$, say $g_{1}=\left(\begin{array}{cc}\cos (t) & -\sin (t) \\ \sin (t) & \cos (t)\end{array}\right)$ with $t \in \mathbb{R}$ (so that $\operatorname{Ad}\left(g_{1}\right)$ corresponds to multiplication with the complex number $\left.\mu:=e^{i t}\right)$, we have

$$
A_{0} \circ \operatorname{Ad}(g)=\mu^{-2} \cdot \operatorname{Ad}(g) \circ A_{0} .
$$

This equation shows that the object which is $\operatorname{Ad}(K)$-invariant and therefore geometrically relevant is not the real structure $A_{0}$ by itself, but rather the "circle of real structures"

$$
\mathfrak{A}_{p_{0}}:=\left\{\lambda A_{0} \mid \lambda \in S^{1}\right\} \text {. }
$$

$\mathfrak{A}_{p_{0}}$ is $\operatorname{Ad}(K)$-invariant and therefore generates an $\operatorname{Ad}(G)$-invariant $S^{1}$-subbundle $\mathfrak{A}$ of the endomorphism bundle $\operatorname{End}\left(T Q^{m *}\right)$, consisting of real structures (conjugations) on the tangent spaces of $Q^{m *}$. For any $A \in \mathfrak{A}$, the tangent line to the fibre of $\mathfrak{A}$ through $A$ is spanned by $J A$.

For any $p \in Q^{m *}$ and $A \in \mathfrak{A}_{p}$, the real structure $A$ induces a splitting 


$$
T_{p} Q^{m *}=V(A) \oplus J V(A)
$$

into two orthogonal, maximal totally real subspaces of the tangent space $T_{p} Q^{m *}$. Here $V(A)$ resp. $J V(A)$ are the $(+1)$-eigenspace resp. the (-1)-eigenspace of $A$. For every unit vector $Z \in T_{p} Q^{m *}$ there exist $t \in\left[0, \frac{\pi}{4}\right], A \in \mathfrak{A}_{p}$ and orthonormal vectors $X, Y \in V(A)$ so that

$$
Z=\cos (t) \cdot X+\sin (t) \cdot J Y
$$

holds; see [18, Proposition 3]. Here $t$ is uniquely determined by $Z$. The vector $Z$ is singular, i.e. contained in more than one Cartan subalgebra of $\mathfrak{m}$, if and only if either $t=0$ or $t=\frac{\pi}{4}$ holds. The vectors with $t=0$ are called $\mathcal{A}$-principal, whereas the vectors with $t=\frac{\pi}{4}$ are called $\mathfrak{A}$-isotropic. If $Z$ is regular, i.e. $0<t<\frac{\pi}{4}$ holds, then also $A$ and $X, Y$ are uniquely determined by $Z$.

Like for the complex quadric, the Riemannian curvature tensor $R$ of $Q^{m *}$ can be fully described in terms of the "fundamental geometric structures" $g, J$ and $\mathfrak{A}$. In fact, under the correspondence $T_{p_{0}} Q^{m *} \cong \mathfrak{m}$, the Riemannian curvature tensor $\bar{R}(X, Y) Z$ corresponds to $-[[X, Y], Z]$ for $X, Y, Z \in \mathfrak{m}$, see [11, Chapter XI, Theorem 3.2(1)]. By evaluating the latter expression explicitly, one can show that one has

$$
\begin{aligned}
\bar{R}(X, Y) Z= & -g(Y, Z) X+g(X, Z) Y \\
& -g(J Y, Z) J X+g(J X, Z) J Y+2 g(J X, Y) J Z \\
& -g(A Y, Z) A X+g(A X, Z) A Y \\
& -g(J A Y, Z) J A X+g(J A X, Z) J A Y
\end{aligned}
$$

for arbitrary $A \in \mathfrak{A}_{p_{0}}$. Therefore the curvature of $Q^{m *}$ is the negative of that of the complex quadric $Q^{m}$, compare [18, Theorem 1]. This confirms that the symmetric space $Q^{m *}$ which we have constructed here is indeed the non-compact dual of the complex quadric.

As Nomizu [12, Theorem 15.3] has shown, there exists one and only one torsionfree covariant derivative $\bar{\nabla}$ on $Q^{m *}$ so that the symmetric involutions $s_{p}: Q^{m *} \rightarrow Q^{m *}$ at $p \in Q^{m *}$ are all affine. $\bar{\nabla}$ is the canonical covariant derivative of $Q^{m *}$. With respect to $\bar{\nabla}$, the action of any member of $G$ on $Q^{m *}$ is also affine. Moreover, $\bar{\nabla}$ is the Levi-Civita connection corresponding to the Riemannian metric $g$, and therefore $g$ is parallel with respect to $\bar{\nabla}$. Moreover, it is well-known that $Q^{m *}$ becomes a Kähler manifold in this way, i.e. the complex structure $J$ is also parallel. Finally, because the $S^{1}$-subbundle $\mathfrak{A}$ of the endomorphism bundle $\operatorname{End}\left(T Q^{m *}\right)$ is $\operatorname{Ad}(G)$-invariant, it is also parallel with respect to the covariant derivative $\bar{\nabla}^{\text {End }}$ induced by $\bar{\nabla}$ on $\operatorname{End}\left(T Q^{m *}\right)$. Because the tangent line of the fiber of $\mathfrak{A}$ through some $A_{p} \in \mathfrak{A}$ is spanned by $J A_{p}$, this means precisely that for any section $A$ of $\mathfrak{U}$ there exists a real-valued 1 -form $q: T Q^{m *} \rightarrow \mathbb{R}$ so that

$$
\bar{\nabla}_{v}^{\text {End }} A=q(v) \cdot J A_{p} \quad \text { holds for } p \in Q^{m *}, v \in T_{p} Q^{m *} .
$$

From the presentation (2.1) of the curvature tensor it follows analogously as for $Q^{m}$ in [18, Sects. 5 and 6] that $Q^{m *}$ has rank 2, that a linear subspace $\mathfrak{a} \subset \mathfrak{m}$ is a Cartan subalgebra if and only if there exist $A \in \mathfrak{A}$ and orthonormal vectors $X, Y \in V(A)$ so that $\mathfrak{a}=\mathbb{R} X \oplus \mathbb{R} J Y$ holds, and that the positive root system $\Sigma^{+}=\left\{\lambda_{1}, \cdots, \lambda_{4}\right\}$ (in terms of the vectors $\lambda_{k}^{\sharp} \in \mathfrak{a}$ dual to the roots $\lambda_{k} \in \mathfrak{a}^{*}$ ) and the corresponding root spaces $\mathfrak{m}_{\lambda_{k}}$ are given by 


\begin{tabular}{lll}
\hline$k$ & $\lambda_{k}^{\#}$ & $\mathfrak{m}_{\lambda_{k}}$ \\
\hline 1 & $\sqrt{2} \cdot J Y$ & $J(V(A) \ominus \mathbb{R} X \ominus \mathbb{R} Y)$ \\
2 & $\sqrt{2} \cdot X$ & $V(A) \ominus \mathbb{R} X \ominus \mathbb{R} Y$ \\
3 & $\sqrt{2} \cdot(X-J Y)$ & $\mathbb{R}(J X+Y)$ \\
4 & $\sqrt{2} \cdot(X+J Y)$ & $\mathbb{R}(J X-Y)$ \\
\hline
\end{tabular}

\section{Tubes around the totally geodesic submanifolds $Q^{m-1 *} \subset Q^{m *}$ and $\mathbb{R} H^{m} \subset Q^{m *}$}

At first we let $P$ be any submanifold of a Riemannian symmetric space $\bar{M}$, and for $p \in P$ we let $\perp_{p} P$ be the normal space of $P$ in $\bar{M}$ at $p$, and let $\perp_{p}^{1} P:=\left\{v \in \perp_{p} P \mid\|v\|=r\right\}$ be the unit sphere in $\perp_{p} P$. We let $\perp P$ resp. $\perp^{r} P$ be the vector bundle resp. the sphere bundle of normal vectors resp. of unit length normal vectors over $P$, and let $\tau: \perp P \rightarrow P$ be the bundle projection map. Moreover, we let $K^{\perp}: T(\perp P) \rightarrow \perp P$ be the normal connection map of $\perp P$, i.e. $K^{\perp} u=\nabla_{u} \mathrm{id}_{\perp P}$. For any $\eta \in \perp P$,

$$
T_{\eta}(\perp P) \rightarrow T_{\tau(\eta)} P \oplus \perp_{\tau(\eta)} P, u \mapsto\left(\tau_{*} u, K^{\perp} u\right)
$$

is then an isomorphism of vector spaces.

The tube map of radius $r>0$ is the map

$$
\Phi: \perp^{1} P \rightarrow \bar{M}, \eta \mapsto \exp _{p}^{\bar{M}}(r \eta),
$$

where $\exp ^{\bar{M}}$ denotes the (geodesic) exponential map of $\bar{M}$. If $\Phi$ is a diffeomorphism into $\bar{M}$, we call its image $M$ the tube around $P$ of radius $r$, it is then a real hypersurface of $\bar{M}$. Berndt [1, Corollary 4.4] has described how to calculate the eigenvalues and eigenvectors of the shape operator of the map $\Phi$, and hence the principal curvatures, their multiplicities and the corresponding principal curvature directions of the tube $M$. We describe his results here only for the case where the submanifold $P$ is totally geodesic.

Therefore suppose that $P$ is a totally geodesic submanifold of $\bar{M}$. Let $\bar{R}$ be the Riemannian curvature tensor of $\bar{M}$. For any $p \in P, \eta \in \perp_{p} P$ we let $\bar{R}_{\eta}:=\bar{R}(\cdot, \eta) \eta$ be the corresponding Jacobi operator of $\bar{M}$ and define $R_{\eta}:=\bar{R}_{\eta} \mid T_{p} P$. Let $\gamma_{\eta}$ be the unit speed geodesic of $\bar{M}$ with $\gamma_{\eta}(0)=p$ and $\dot{\gamma}_{\eta}(0)=\eta$; then $\gamma_{\eta}(r)=\Phi(p) \in M$ and $\dot{\gamma}_{\eta}(r)$ is a unit normal vector of $M$; we say that $\dot{\gamma}_{\eta}(r)$ is pointing outward and that $-\dot{\gamma}_{\eta}(r)$ is pointing inward. For the description of the principal curvatures, we follow [1, Sect. 4.2] by considering for any $\kappa \in \mathbb{R}$ the solution functions $\sin _{\kappa}$ and $\cos _{\kappa}$ of the second order differential equation $y^{\prime \prime}+\kappa y=0$ with $\sin _{\kappa}(0)=0, \sin _{\kappa}^{\prime}(0)=1$ and $\cos _{\kappa}(0)=1, \cos _{\kappa}^{\prime}(0)=0$. Explicitly one has

$$
\sin _{\kappa}(t)=\left\{\begin{array}{ll}
\frac{1}{\sqrt{\kappa}} \sin (\sqrt{\kappa} t) & \text { for } \kappa>0 \\
t & \text { for } \kappa=0 \\
\frac{1}{\sqrt{-\kappa}} \sinh (\sqrt{-\kappa} t) & \text { for } \kappa<0
\end{array} \quad \text { and } \quad \cos _{\kappa}(t)=\left\{\begin{array}{ll}
\cos (\sqrt{\kappa} t) & \text { for } \kappa>0 \\
1 & \text { for } \kappa=0 \\
\cosh (\sqrt{-\kappa} t) & \text { for } \kappa<0
\end{array} .\right.\right.
$$

According to [1, Corollary 4.4], the shape operator $S^{\Phi}$ with respect to the outward-pointing unit normal vector $\dot{\gamma}(r)$ has two types of eigenvalues, and any eigenvalue or eigenvector of $S^{\Phi}$ is obtained by either of these two methods: 
(I) For any eigenvalue $\kappa$ of $R_{\eta}$, the number $\kappa \cdot \frac{\sin _{\kappa}(r)}{\cos _{\kappa}(r)}$ is an eigenvalue of $S^{\Phi}$. The corresponding eigenspace of $S^{\Phi}$ is given by

$$
\left\{u \in T_{\eta}\left(\perp^{1} P\right) \mid \tau_{*} u \in E_{\kappa}, K^{\perp} u=0\right\},
$$

where $E_{\kappa}$ denotes the eigenspace of $R_{\eta}$ for the eigenvalue $\kappa$.

(II) For any eigenvalue $\kappa$ of $\bar{R}_{\eta} \mid\left(\perp_{\tau(\eta)} P \ominus \mathbb{R} \eta\right)$, the number $-\frac{\cos _{\kappa}(r)}{\sin _{\kappa}(r)}$ is an eigenvalue of $S^{\Phi}$. The corresponding eigenspace of $S^{\Phi}$ is given by

$$
\left\{u \in T_{\eta}\left(\perp^{1} P\right) \mid \tau_{*} u=0, K^{\perp} u \in \bar{E}_{\kappa}\right\},
$$

where $\bar{E}_{\kappa}$ denotes the eigenspace of $\bar{R}_{\eta} \mid\left(\perp_{\tau(\eta)} P \ominus \mathbb{R} \eta\right)$ for the eigenvalue $\kappa$.

The principal curvatures of $M$ at $\Phi(\eta)$ are precisely the eigenvalues of $S^{\Phi}$ described above. The corresponding principal curvature spaces are the image under $\Phi_{*}$ of the eigenspaces of $S^{\Phi}$; hence, they are obtained from the eigenspaces $E_{\kappa}$ (in the case (I)) resp. $\bar{E}_{\kappa}$ (in the case (II)) by parallel transport along the geodesic $\gamma_{\eta}$.

We will now apply the preceding results to tubes in the complex hyperbolic quadric $\bar{M}=Q^{m *}$ around the two types of totally geodesic submanifolds that occur in Theorem A(i),(iii). It follows from Eq. (2.1) that if the unit normal vector $\eta \in \perp^{1} Q^{m *}$ is $\mathfrak{A}$-principal, the corresponding Jacobi operator is given by

$$
\bar{R}_{\eta}(X)=-X-A X+2 g(X, \eta) \eta-2 g(X, J \eta) J \eta .
$$

It is easy to see that $\bar{R}_{\eta}$ then has the two eigenvalues 0 and -2 . If $A \in \mathfrak{A}$ is such that $\eta \in V(A)$ holds, then the corresponding eigenspaces are $\mathbb{R} \eta \oplus J(V(A) \ominus \mathbb{R} \eta)$ and $(V(A) \ominus \mathbb{R} \eta) \oplus \mathbb{R} J \eta$, respectively.

The obvious embedding of Lie groups $\mathrm{SO}_{2, m-1}^{o} \rightarrow S O_{2, m}^{o}$ induces a totally geodesic embedding of $Q^{m-1^{*}}=\mathrm{SO}_{2, m-1} / \mathrm{SO}_{2} \mathrm{SO}_{m-1}$ into $Q^{m *}=S \mathrm{O}_{2, m} / \mathrm{SO}_{2} \mathrm{SO}_{m}$. We will view $Q^{m-1 *}$ as a totally geodesic complex hypersurface of $Q^{m *}$ by means of this embedding.

Proposition 3.1 The tube $M$ around the totally geodesic $Q^{m-1^{*}}$ in $Q^{m *}$ exists for every radius $r>0$. For $M$ the following statements hold:

(1) Every normal vector $N$ of $M$ is $\mathfrak{A}$-principal.

(2) $M$ has constant principal curvatures, and in particular constant mean curvature. Let $N$ be the outward-pointing unit normal vector of $M$ at $q \in M$. By (1) we have $N \in V(A)$ for some $A \in \mathfrak{A}_{q}$. Then the principal curvatures of $M$ with respect to $N$ and the corresponding principal curvature spaces are

\begin{tabular}{lll}
\hline Principal curvature & Principal curvature space & Multiplicity \\
\hline$\lambda=0$ & $J(V(A) \ominus \mathbb{R} N)$ & $m-1$ \\
$\mu=-\sqrt{2} \tanh (\sqrt{2} r)$ & $V(A) \ominus \mathbb{R} N$ & $m-1$ \\
$\alpha=-\sqrt{2} \operatorname{coth}(\sqrt{2} r)$ & $\mathbb{R} J N$ & 1 \\
\hline
\end{tabular}

(3) $M$ is a Hopf hypersurface.

(4) The shape operator $S$ and the structure tensor field $\phi\left(\phi=\operatorname{pr}_{T M} \circ J\right.$, where $\mathrm{pr}_{T M}: T Q^{m *} \rightarrow T M$ denotes the orthogonal projection) satisfy 


$$
S \phi+\phi S=\mu \cdot \phi .
$$

In particular $M$ is a contact submanifold.

Proof It follows from the construction of the $S^{1}$-subbundle $\mathfrak{A}$ of $\operatorname{End}\left(T Q^{m *}\right)$ of real structures that for any $p \in Q^{m-1^{*}}$, both complex subspaces $T_{p} Q^{m-1^{*}}$ and $\perp_{p} Q^{m-1^{*}}$ are invariant under every $A_{p} \in \mathfrak{A}_{p}$. Because $\perp_{p} Q^{m-1^{*}}$ thus is a complex one-dimensional subspace that is invariant under $A_{p} \in \mathfrak{A}_{p}$, it follows that the vectors in this space are $\mathfrak{A}$-principal.

Now let $q \in M$ be given. It follows from the construction of the tube $M$ that there exists $p \in Q^{m-1 *}$ and a unit normal vector $\eta \in \perp_{p} Q^{m-1 *}$ so that the normal space $\perp_{q} M$ of $M$ at $q$ is spanned by $\dot{\gamma}_{\eta}(r)$. Because $\dot{\gamma}_{\eta}(0) \in \perp_{p} Q^{m-1^{*}}$ is $\mathfrak{A}$-principal by the preceding observation, and the $S^{1}$-bundle $\mathfrak{A}$ is parallel, it follows that also $\dot{\gamma}_{\eta}(r)$ and hence $\perp_{q} M$ is $\mathfrak{A}$-principal.

Moreover the outward-pointing unit normal vector $N$ of $M$ at $q$ equals $\dot{\gamma}_{\eta}(r)$ and is contained in $V\left(A_{q}\right)$, where $A_{q} \in \mathfrak{A}_{q}$ is the parallel transport of $A_{p}$ along the geodesic $\gamma_{\eta}$. Note that $T_{p} Q^{m-1 *^{q}}=\left(V\left(A_{p}\right) \ominus \mathbb{R} \eta\right) \oplus J\left(V\left(A_{p}\right) \ominus \mathbb{R} \eta\right)$ and $\perp_{p} Q^{m-1 *}=\mathbb{R} \eta \oplus \mathbb{R} J \eta$ holds. We now apply the two cases (I) and (II) given above to obtain the principal curvatures of $M$ with respect to $N$. For case (I), we note that $R_{\eta}:=\bar{R}_{\eta} \mid T_{p} Q^{m-1^{*}}$ has the two eigenvalues 0 and -2 , with eigenspaces $J\left(V\left(A_{p}\right) \ominus \mathbb{R} \eta\right)$ and $V\left(A_{p}\right) \ominus \mathbb{R} \eta$, respectively. From the eigenvalue $\kappa=0$ we obtain the principal curvature $\kappa \cdot \frac{\sin _{\kappa}(r)}{\cos _{\kappa}(r)}=0=\lambda$, and from the eigenvalue $\kappa=-2$ we obtain the principal curvature $\kappa \cdot \frac{\sin _{\kappa}(r)}{\cos _{\kappa}(r)}=-\sqrt{2} \tanh (\sqrt{2} r)=\mu$. In case (II), we have $\perp_{p} Q^{m-1^{*}} \ominus \mathbb{R} \eta=\mathbb{R} J \eta$, and therefore the only eigenvalue of $\bar{R}_{\eta} \mid\left(\perp_{p} Q^{m-1^{*}} \ominus \mathbb{R} \eta\right)$ is $\kappa=-2$ with the eigenspace $\mathbb{R} J \eta$. This yields the principal curvature $-\frac{\cos _{\kappa}(r)}{\sin _{\kappa}(r)}=-\frac{\cos _{\kappa}(r)}{\sin _{\kappa}(r)}=-\sqrt{2} \operatorname{coth}(\sqrt{2} r)=\alpha$. The principal curvature spaces are obtained from the corresponding eigenspaces of $\bar{R}_{\eta}$ by parallel transport along $\gamma_{\eta}$ and are therefore as stated in the proposition.

It follows from the calculation of the principal curvatures that $M$ has constant principal curvatures, in particular constant mean curvature. The claim that $M$ is Hopf means by definition that the Hopf vector field $\xi=-J N$ is a principal vector field; this is the case by our previous calculation. The corresponding principal curvature is $\alpha=-\sqrt{2} \operatorname{coth}(\sqrt{2} r)$. Moreover, it is easily seen that $\phi(J N)=0$ holds, and that $\phi$ acts as $J$ on $V\left(A_{q}\right) \ominus \mathbb{R} N$ and on $J\left(V\left(A_{q}\right) \ominus \mathbb{R} N\right)$. Therefore the equation in (4) is easily verified on the principal curvature spaces described in (2). Because Eq. (1.2) thus holds for $M$ with $k=\mu$, it follows that $M$ is a contact submanifold.

A similar discussion applies to tubes around the totally geodesic $\mathbb{R} H^{m}$ in $Q^{m *}$. The obvious embedding of Lie groups $\mathrm{SO}_{1, m} \rightarrow \mathrm{SO}_{2, m}$ induces a totally geodesic embedding of $\mathbb{R} H^{m}=S O_{1, m} / S O_{m}$ into $Q^{m *}=S O_{2, m} / S O_{2} S O_{m}$. We will view $\mathbb{R} H^{m}$ as a real form, i.e. a totally geodesic, totally real, real-m-dimensional submanifold of $Q^{m *}$ by means of this embedding.

Proposition 3.2 The tube $M$ around the totally geodesic $\mathbb{R} H^{m}$ in $Q^{m *}$ exists for every radius $r>0$. For $M$ the following statements hold:

(1) Every normal vector $N$ of $M$ is $\mathfrak{A}$-principal.

(2) $M$ has constant principal curvatures, and in particular constant mean curvature. Let $N$ be the outward-pointing unit normal vector of $M$ at $q \in M$. By (1) we have $N \in V(A)$ for some $A \in \mathfrak{A}_{q}$. Then the principal curvatures of $M$ with respect to $N$ and the corresponding principal curvature spaces are 


\begin{tabular}{lll}
\hline Principal curvature & Principal curvature space & Multiplicity \\
\hline$\lambda=0$ & $J(V(A) \ominus \mathbb{R} N)$ & $m-1$ \\
$\mu=-\sqrt{2} \operatorname{coth}(\sqrt{2} r)$ & $V(A) \ominus \mathbb{R} N$ & $m-1$ \\
$\alpha=-\sqrt{2} \tanh (\sqrt{2} r)$ & $\mathbb{R} J N$ & 1 \\
\hline
\end{tabular}

(3) $M$ is a Hopf hypersurface.

(4) The shape operator $S$ and the structure tensor field $\phi\left(\phi=\operatorname{pr}_{T M} \circ J\right.$, where $\mathrm{pr}_{T M}: T Q^{m *} \rightarrow T M$ denotes the orthogonal projection) satisfy

$$
S \phi+\phi S=\mu \cdot \phi .
$$

In particular $M$ is a contact submanifold.

Proof The proof of this proposition follows the same pattern as that of Proposition 3.1.

For any $p \in \mathbb{R} H^{m}$, there exists $A_{p} \in \mathfrak{A}_{p}$ so that $T_{p} \mathbb{R} H^{m}=V\left(-A_{p}\right)$ and therefore $\perp_{p} \mathbb{R} H^{m}=J V\left(-A_{p}\right)=V\left(A_{p}\right)$ holds. In particular, both $T_{p} \mathbb{R} H^{m}$ and $\perp_{p} \mathbb{R} H^{m}$ are $\mathfrak{A}$-principal. Now let $q \in M$ be given. Again there exists $p \in \mathbb{R} H^{m}$ and $\eta \in \perp_{p}^{1} \mathbb{R} H^{m}$ so that $\perp_{q} M$ is spanned by $\dot{\gamma}_{\eta}(r)$. Because $\dot{\gamma}_{\eta}(0) \in \perp_{p} \mathbb{R} H^{m}=V\left(A_{p}\right)$ is $\mathfrak{A}$-principal, it again follows that also $\dot{\gamma}_{\eta}(r)$ and hence $\perp_{q} M$ is $\mathfrak{A}$-principal. Hence the outward-pointing unit normal vector $N=\gamma_{\eta}(r)$ is contained in $V\left(A_{q}\right)$ where $A_{q} \in \mathfrak{A}_{q}$ is the parallel transport of $A_{p}$ along $\gamma_{\eta}$.

We again apply the two cases (I) and (II) given above to obtain the principal curvatures of $M$ with respect to $N$. For case (I), we note that $R_{\eta}:=\bar{R}_{\eta} \mid T_{p} \mathbb{R} H^{m}$ has the two eigenvalues 0 and -2 with eigenspaces $J\left(V\left(A_{p}\right) \ominus \mathbb{R} \eta\right)$ and $\mathbb{R} J \eta$, respectively. From the eigenvalue $\kappa=0$ we obtain $\kappa \cdot \frac{\sin _{\kappa}(r)}{\cos _{\kappa}(r)}=0=\lambda$, and from the eigenvalue $\kappa=-2$ we obtain the principal curvature $\quad \kappa \cdot \frac{\sin _{\kappa}(r)}{\cos _{\kappa}(r)}=-\sqrt{2} \tanh (\sqrt{2} r)=\alpha$. In case (II), we have $\perp_{p} \mathbb{R} H^{m} \ominus \mathbb{R} \eta=V\left(A_{p}\right) \ominus \mathbb{R} \eta$, and therefore the only eigenvalue of $\bar{R}_{\eta} \mid\left(\perp_{p} Q^{m-1^{*}} \ominus \mathbb{R} \eta\right)$ is $\kappa=-2$ with the eigenspace $V\left(A_{p}\right) \ominus \mathbb{R} \eta$. This yields the principal curvature $-\frac{\cos _{\kappa}(r)}{\sin _{\kappa}(r)}=-\frac{\cos _{\kappa}(r)}{\sin _{\kappa}(r)}=-\sqrt{2} \operatorname{coth}(\sqrt{2} r)=\mu$.

The remaining parts of the proposition now follow in the same way as in the proof of Proposition 3.1.

\section{Horospheres in $Q^{m *}$}

Suppose that $\bar{M}$ is a Hadamard manifold, i.e. a simply connected, complete Riemannian manifold with sectional curvature $\leq 0$. We denote by $d$ the Riemannian distance function on $\bar{M}$. Two unit speed geodesics $\gamma_{1}, \gamma_{2}: \mathbb{R} \rightarrow \bar{M}$ are said to be asymptotic to each other, if the function $t \mapsto d\left(\gamma_{1}(t), \gamma_{2}(t)\right)$ remains bounded for $t \rightarrow \infty$. Asymptoticness defines an equivalence relation on the space of unit speed geodesics on $\bar{M}$. The equivalence classes are called points at infinity, and their set is denoted by $\bar{M}(\infty)$. For any unit speed geodesic $\gamma: \mathbb{R} \rightarrow \bar{M}$, the corresponding point at infinity is denoted by $\gamma(\infty) \in \bar{M}(\infty)$. The horosphere with center at infinity $\gamma(\infty)$ through some point $p \in \bar{M}$ is defined as

$$
C(p, \gamma(\infty))=\left\{\begin{array}{l|l}
q \in \bar{M} & \lim _{t \rightarrow \infty}(d(q, \gamma(t))-d(p, \gamma(t)))=0
\end{array}\right\} .
$$


It can be shown that $C(p, \gamma(\infty))$ indeed does not depend on the choice of the representant $\gamma$ within the equivalence class $\gamma(\infty)$ and that it is a real hypersurface of $\bar{M}$, see [8, Sect. 1.10].

We now suppose that $\bar{M}$ is a Riemannian symmetric space of non-compact type and rank 2. The following construction principle for horospheres in $\bar{M}$ was described by Berndt and Suh in [3, Sect. 2]. Let us consider $\bar{M}=G / K$ with the "origin" $o:=e K \in \bar{M}$, the Cartan decomposition $\mathfrak{g}=\mathfrak{f} \oplus \mathfrak{m}$ and a Cartan subalgebra $\mathfrak{a} \subset \mathfrak{m}$. Further consider the root system $\Sigma \subset \mathfrak{a}^{*}$ and the corresponding root space decomposition $\mathfrak{g}=\mathfrak{a} \oplus \mathfrak{f}_{\mathfrak{a}} \oplus \bigoplus_{\lambda \in \Sigma} \mathfrak{g}_{\lambda}$. For a positive root system $\Sigma^{+} \subset \Sigma, \mathfrak{n}:=\bigoplus_{\lambda \in \Sigma^{+}} \mathfrak{g}_{\lambda}$ is a nilpotent subalgebra of $\mathfrak{g}$, and $\mathfrak{g}=\mathfrak{f} \oplus \mathfrak{a} \oplus \mathfrak{n}$ is an Iwasawa decomposition of $\mathfrak{g}$. Let $A$ and $N$ be the connected Lie subgroups of $G$ with Lie algebra $a$ resp. $\mathfrak{n}$. Then $G=K A N$ is an Iwasawa decomposition of $G$, more precisely, $A$ and $N$ are simply connected, $K \cap A N=\{e\}$ holds, and the maps

$$
K \times A \times N \rightarrow G,(k, a, n) \mapsto k a n \quad \text { and } A \times N \rightarrow \bar{M},(a, n) \mapsto a n \cdot o
$$

are surjective diffeomorphisms, see [10, Theorem VI.6.46, p. 374].

Now suppose that a unit vector $H \in \mathfrak{a}$ is given. Then $\mathfrak{g}_{H}:=(\mathfrak{a} \ominus \mathbb{R} H) \oplus \mathfrak{n}$ is a solvable Lie subalgebra of $\mathfrak{g}$. Let $S_{H}$ be the connected subgroup of $A N$ with Lie algebra $\mathfrak{g}_{H}$. Then the orbits of the action of $S_{H}$ on $\bar{M}$ are the horospheres of $\bar{M}$ with the center at infinity $\gamma_{H}(\infty)$, where $\gamma_{H}$ is the geodesic with $\gamma_{H}(0)=o$ and $\dot{\gamma}_{H}(0)=H$ (and where we identify $\mathfrak{m}$ with $T_{e} \bar{M}$ in the usual manner). In particular we have $C\left(o, \gamma_{H}(\infty)\right)=S_{H} \cdot o$. It was shown by Berndt and Tamaru in [5, Proposition 3.1(2),(3)] that the shape operator of $C\left(o, \gamma_{H}(\infty)\right)$ with respect to the unit normal vector $H$ is given by $\operatorname{ad}(H) \mid \mathfrak{s}_{H}$. Therefore the principal curvatures are constant, and their values are given by 0 and by $\lambda(H)$ for every $\lambda \in \Sigma^{+}$. The corresponding principal curvature spaces are $\mathfrak{a} \ominus \mathbb{R} H$ and $\mathfrak{g}_{\lambda}$; under the standard identification $T_{e} \bar{M} \cong \mathfrak{m}$, the latter space corresponds to $\mathfrak{m}_{\lambda}=\left\{X-\theta X \mid X \in \mathfrak{g}_{\lambda}\right\}$ (where $\theta: \mathfrak{g} \rightarrow \mathfrak{g}$ denotes the Cartan involution).

We will now apply this construction to $\bar{M}=Q^{m *}$ and an $\mathfrak{A}$-principal vector $H \in \mathfrak{a}$.

Proposition 4.1 Let $M$ be a horosphere in $Q^{m *}$ with its center at infinity being given by an $\mathfrak{A}$-principal geodesic $\gamma$. Then the following statements hold:

(1) Every normal vector $N$ of $M$ is $\mathfrak{A}$-principal.

(2) $M$ has constant principal curvatures, and in particular constant mean curvature. Then the principal curvatures of $M$ with respect to the unit normal vector ${ }^{1} N:=-\dot{\gamma}(0)$ and the corresponding principal curvature spaces are

\begin{tabular}{lll}
\hline Principal curvature & Principal curvature space & Multiplicity \\
\hline 0 & $J(V(A) \ominus \mathbb{R} N)$ & $m-1$ \\
$-\sqrt{2}$ & $(V(A) \ominus \mathbb{R} N) \oplus \mathbb{R} J N$ & $m$ \\
\hline
\end{tabular}

Here $A \in \mathfrak{A}$ is chosen such that $-\dot{\gamma}(0) \in V(A)$ holds.

(3) $M$ is a Hopf hypersurface.

(4) The shape operator $S$ and the structure tensor field $\phi\left(\phi=\mathrm{pr}_{T M} \circ J\right.$, where $\mathrm{pr}_{T M}: T Q^{m *} \rightarrow T M$ denotes the orthogonal projection) satisfy

\footnotetext{
1 We choose the negative of $\dot{\gamma}(0)$ as normal vector here so that the orientation matches the one considered for the tubes in Sect. 3 .
} 


$$
S \phi+\phi S=-\sqrt{2} \cdot \phi .
$$

In particular $M$ is a contact submanifold.

Proof We use the description of the Cartan subalgebras, the roots and the root spaces of $Q^{m *}$ given at the end of Sect. 2. We may assume without loss of generality that $\gamma(0)=o$ and $\dot{\gamma}(0) \in \mathfrak{a}$ holds. Because the geodesic $\gamma$ is $\mathfrak{A}$-principal, there exists $A \in \mathfrak{A}_{o}$ with $X:=\dot{\gamma}(0) \in V(A)$, and we may further assume that the Cartan subalgebra $\mathfrak{a}$ is given by $\mathfrak{a}=\mathbb{R} X \oplus \mathbb{R} J Y$ with a unit vector $Y \in V(A) \ominus \mathbb{R} X$. Numbering the positive roots of $Q^{m *}$ as in Sect. 2 we then have

$$
\lambda_{1}(-X)=0 \quad \text { and } \quad \lambda_{2}(-X)=\lambda_{3}(-X)=\lambda_{4}(-X)=-\sqrt{2} .
$$

It follows that the horosphere $M$ has the two principal curvatures 0 with principal curvature space $(\mathfrak{a} \ominus \mathbb{R} X) \oplus \mathfrak{m}_{\lambda_{1}}=J(V(A) \ominus \mathbb{R} X)$, and $-\sqrt{2}$ with the principal curvature space $\mathfrak{m}_{\lambda_{2}} \oplus \mathfrak{m}_{\lambda_{3}} \oplus \mathfrak{m}_{\lambda_{4}}=(V(A) \ominus \mathbb{R} X) \oplus \mathbb{R} J X$.

The remainder of the statements follows in the same way as for the proofs of Sect. 3.

Remark 4.2 Note that for both the family of tubes around $Q^{m-1 *}$ (Proposition 3.1) and the family of tubes around $\mathbb{R} H^{m}$ (Proposition 3.2), when one lets the radius $r>0$ of the tube tend to infinity, the values of the principal curvatures and the corresponding principal curvature spaces tend to the values and spaces of the horosphere with $\mathfrak{A}$-principal center at infinity (Proposition 4.1). In this sense, this horosphere is the joint limit of both mentioned families of tubes as $r \rightarrow \infty$.

Acknowledgements This work was done while Sebastian Klein was visiting professor at the Research Institute of Real and Complex Submanifolds in Kyungpook National University during October, 2017.

\section{References}

1. Berndt, J.: Über Untermannigfaltigkeiten von komplexen Raumformen, Ph.D. thesis, Universität zu Köln (1989)

2. Berndt, J., Lee, H., Suh, Y.J.: Contact hypersurfaces in noncompact complex Grassmannians of rank two. Int. J. Math. 24(11), 1350089 (2013)

3. Berndt, J., Suh, Y.J.: Hypersurfaces in noncompact complex Grassmannians of rank two. Int. J. Math. 23(35), 1250103 (2012)

4. Berndt, J., Suh, Y.J.: Contact hypersurfaces in Kähler manifolds. Proc. Am. Math. Soc. 143, 26372649 (2015)

5. Berndt, J., Tamaru, H.: Homogeneous codimension one foliations on noncompact symmetric spaces. J. Differ. Geom. 63, 1-40 (2003)

6. Blair, D.E.: Riemannian Geometry of Contact and Symplectic Manifolds, Progress in Mathematics. Springer, Berlin (2010)

7. Dragomir, S., Perrone, D.: Harmonic Vector Fields: Variational Principles and Differential Geometry. Elsevier, New York (2011)

8. Eberlein, P.: Geometry of Nonpositively Curved Manifolds. The University of Chicago Press, Chicago (1996)

9. Klein, S.: Totally geodesic submanifolds in the complex quadric. Differ. Geom. Appl. 26, 79-96 (2008)

10. Knapp, A.W.: Lie Groups Beyond an Introduction, Progress in Mathematics. Birkhäuser, Basel (2002) 
11. Kobayashi, S., Nomizu, K.: Foundations of Differential Geometry, vol. II, Wiley Classics Library edn. Wiley, New York (1996)

12. Nomizu, K.: Invariant affine connections on homogeneous spaces. Am. J. Math. 76, 33-65 (1954)

13. Okumura, M.: Contact hypersurfaces in certain Kählerian manifolds. Tôhoku Math. J. 18, 74-102 (1966)

14. Pérez, J.D.: Commutativity of Cho and structure Jacobi operators of a real hypersurface in a complex projective space. Ann. di Mat. Pure Appl. 194, 1781-1794 (2015)

15. Pérez, J.D., Jeong, I., Ko, J., Suh, Y.J.: Real hypersurfaces with Killing shape operator in the complex quadric. Mediterr. J. Math. 15(1), 15 (2018)

16. Pérez, J.D., Suh, Y.J.: Real hypersurfaces in complex two-plane Grassmannians with parallel and commuting Ricci tensor. J. Korean Math. Soc. 44, 211-235 (2007)

17. Pérez, J.D., Suh, Y.J., Watanabe, Y.: Generalized Einstein real hypersurfaces in complex two-plane Grassmannians. J. Geom. Phys. 60, 1806-1818 (2010)

18. Reckziegel, H.: On the geometry of the complex quadric. In: Dillen F, Komrakov B, Simon U, Van de Woestyne I, Verstraelen L (eds.) Geometry and Topology of Submanifolds VIII (Brussels/Nordfjordeid 1995), World Scientific Publishing, River Edge, pp. 302-315 (1995)

19. Sasaki, S.: On differentiable manifolds with certain structures which are closely related to almost contact structure, I. Tôhoku Math. J. 12, 459-476 (1960)

20. Smyth, B.: Homogeneous complex hypersurfaces. J. Math. Soc. Jpn. 20, 643-647 (1968)

21. Suh, Y.J.: Real hypersurfaces of type B in complex two-plane Grassmannians. Monatsh. Math. 147, 337-355 (2006)

22. Suh, Y.J.: Contact Real Hypersurfaces in the Complex Quadric, submitted for publication, p. 22 (2017)

23. Vernon, M.H.: Contact hypersurfaces of a complex hyperbolic space. Tôhoku Math. J. 39, 215-222 (1987)

24. Yano, K., Kon, M.: CR submanifolds in Kählerian and Sasakian manifolds. Progress in Mathematics. Birkhäuser, Boston (1983)

Publisher's Note Springer Nature remains neutral with regard to jurisdictional claims in published maps and institutional affiliations. 